\title{
Applications of Nuclear and Particle Physics Technology: Particles \& Detection - A Brief Overview
}

\author{
Andrew G. Weisenberger \\ Thomas Jefferson National Accelerator Facility \\ 12000 Jefferson Avenue Newport News, Virginia, 23606, USA \\ drew@jlab.org
}

Published 3 May 2018

\begin{abstract}
A brief overview of the technology applications with significant societal benefit that have their origins in nuclear and particle physics research is presented. It is shown through representative examples that applications of nuclear physics can be classified into two basic areas: 1) applying the results of experimental nuclear physics and 2) applying the tools of experimental nuclear physics. Examples of the application of the tools of experimental nuclear and particle physics research are provided in the fields of accelerator and detector based technologies namely synchrotron light sources, nuclear medicine, ion implantation and radiation therapy.
\end{abstract}

Keywords: accelerators; detectors; radiation; bio-medical.

\section{Introduction}

Several sources have described the multitude of applications that have sprung from nuclear particle physics research. The purpose of this summary given the limited space available is not to compete with existing examinations of the contributions of nuclear particle physics research to societal needs. A straightforward taxonomy of the technologies derived from nuclear physics research is presented. It is hoped that representative examples will assist physicists and others to communicate to others that beyond enlarging our basic understanding of the nature of matter, nuclear physics has made and continues to make a big impact on society. More complete descriptions of examples of applications of nuclear physics research can be found in the sources sited. "Industrial Accelerators and Their Applications," "Reviews of Accelerator Science and Technology" and chapter three of the publication prepared by the National Research Council entitled "Nuclear Physics: Exploring the Heart of Matter" are valuable places to start. $^{1-3}$

The primary applications of nuclear particle physics can be divided into two areas: 1) applying the results of experimental nuclear physics and 2) applying the tools of experimental nuclear physics. It is under these headings that such applications as material processing, material analysis, and medicine are some of the examples of society benefiting from nuclear physics research.

This is an Open Access article published by World Scientific Publishing Company. It is distributed under the terms of the Creative Commons Attribution 4.0 (CC-BY) License. Further distribution of this work is permitted, provided the original work is properly cited. 


\section{Applying the Results of Experimental Nuclear Physics}

The knowledge reaped from experimental nuclear physics naturally produces new understanding that leads to new tools that can expand out into various unanticipated applications. One example is our understanding of radioactivity and radioisotopes. This has lead to methods of radioisotope production leading to the development of radiotracer science in which biological molecules can be tagged which radioisotopes to be detectors and/or imaged externally. Radioisotopes are chosen for the task based on the emission of $\mathrm{x}$-rays, gamma-rays, beta particles or positrons. Detection of these emissions is handled by detector technology suited for the goal.

Other examples abound in which nuclear physic research has lead to new understandings such as the physics of the interaction of high energy particles in matter providing us with the understanding of nuclear reactions, ionization, spallation, characteristic x-rays, bremsstrahlung and nuclear activation. Power generation and military applications are clear examples of the application of our understanding of the nuclear reactions of fission and fusion. This understanding of the basic physics has lead to power generation and beyond. Over several decades we have developed great tools to increase our understanding of the nature of the atomic nucleus, the nucleons, quarks and more. I will now focus on how we have leveraged some of the tools of nuclear physics research to societal changing applications.

\section{Applying the Tools of Experimental Nuclear Physics}

\subsection{Accelerator technology used with nuclear physics detector technology}

Technology was developed about hundred years ago designed to probe the structure of matter. In its basic form it is the acceleration of particles (e.g. electrons, $\alpha$-particles, ions etc.) towards some target made up of a foil, liquid or gas. The interaction is reconstructed through a single detector or collection of detectors. The detectors are designed to detect the primary accelerated particle and any other particles that are knocked loose or created. To achieve higher energy thresholds particle accelerators of various sorts such as Van DeGraaff accelerators, Cockcroft-Walton accelerators, cyclotrons, synchrotrons, linear accelerators (linacs) etc. have been developed to accelerate a particle at various kinetic energies to facilitate the probing of matter. This process can also be used to generate collisions between two beams of accelerated particles such as is the case with the Large Hadron Collider in CERN, the world's largest accelerator. High energy particle detectors designed and built based on the understanding of interaction of the resultant particles in the detector material have been developed with names such as scintillators (plastic, crystal, liquid or gas), Cherenkov detectors, multi-wire proportional chambers, solid state detectors, drift chambers, time projection chambers etc.

This arrangement of accelerating "particles" towards some "target" and then detecting/imaging an interaction has been translated from a basic tool of particle physics to several applications with compelling societal impact. Particles such as electrons, protons and ions are directed to a material of interest and the interaction produces a 
scattering (or deflection via external magnetic fields) of the primary particle and the generation of other particles such as photons and neutrons that are used to analyze the material. Special detectors are used to observe the result of the interaction. Neutrons originating from the decay radioisotopes or nuclear reactors have also been used for neutron based studies; however, accelerator generated neutrons offer more control. Examples of typical detector technology brought to bear in these applications include scintillators and phosphors which produce optical photons typically detected by photomultipliers such as microchannel plates (MCP) and charged coupled devices (CCDs). The pairing of a nuclear particle physics accelerator and detector technology in applications beyond nuclear physics continues to grow. Some of these applications include: neutron tomography, neutron diffraction, ion beam analysis, accelerator mass spectrometry, synchrotron light source and medical x-ray radiography/tomography

Since most people have undergone medical x-ray radiography, this application of the tools of nuclear physics is probably the most familiar. Perhaps not so familiar to most in this class of applications is the significant impact of synchrotron light sources.

\subsubsection{Synchrotron Light}

Globally there are nearly 25 synchrotron light sources using particle accelerators built to accelerate charged particles at relativistic velocities in curved paths. The magnetically induced beam curvature produces high energy electromagnetic radiation also known as synchrotron radiation. Because the $\mathrm{x}$-ray photons produced via synchrotron radiation are intense and nearly mono-energetic they can be used to analyze materials at high spatial resolution and on the molecular structure level. X-ray crystallography and high-resolution $\mathrm{x}$-ray imaging are two of the main applications synchrotron light, others include: drug development, cell biology, elemental analysis, toxicology, forensics and historical artifact analysis.

The basic components of synchrotron light sources would be familiar to experimental nuclear physicists. A typical system would include: an electron gun, linear accelerator, booster synchrotron, storage ring, beam lines and experiment stations. As in typical nuclear physics experimental facilities there is a need for detectors to analyze the particles involved in the interaction with the target. Typical detectors include, charge coupled devices (CCD), intensified CCDs, electron multiplying CCDs and gas based detectors. Most synchrotron light sources are facilities that can accommodate industrial as well as academic users. New facilities are being built globally allowing this powerful research tool to continue to make advances spanning several disciplines.

\subsection{Nuclear physics detector technology used without accelerators}

Often just the tool for detecting the high energy particles in nuclear physics experimentation has been brought to bear to fill a need. Examples of such applications of detector technology in this area include: muon radiography / tomography, smoke detectors, gamma-ray spectrometry (radioisotope detection and identification), and molecular imaging (using radiotracers) such as autoradiography, gamma cameras, Single 
Photon Emission Computed Tomography (SPECT) and Positron Emission Tomography (PET). The detectors involved in these applications focus on sensing trace amounts of radioisotopes via their emitted radiation. The primary goals are to quantify the amount and visualize the spatial distribution of the radioisotope undergoing radioactive decay. Beta particles, $\mathrm{x}$-rays, positrons, gamma-rays or alpha particles are the many particles which could be emitted in the decay process. In this class of applications, detector technology with origins in nuclear particle physics is used in conjunction with specific radioisotopes. No accelerator technology is typically used. Detectors can be based on: ion chambers, scintillators/phosphors, photomultipliers or solid-state devices. Because of societal impact I will focus on smoke detectors and molecular imaging.

\subsubsection{Smoke detectors}

In terms of the shear total numbers of units in use under this class of application, the ion chamber based smoke detector is the leader. ${ }^{4}$ The first smoke detector developed and widely used contains the radioisotope Americium-241 which is an alpha particle emitter that was first produced in 1945 at the University of Chicago. It was in 1976 when a United States patent was awarded for a smoke detector based a simple process using Americium-241. The emitted alpha particle ionizes the air molecules between two electrical plates in the top and bottom of a small pill box sized ion chamber that is open to room air. The electrical plates are part of an electrical circuit that can conduct a small current of electricity through the ionized air. If smoke particles enter the chamber the ionization is disturbed and the electrical current is broken causing an electrical circuit to sense the change and sounding an alarm. While it is difficult to measure the economic impact of the discovery of Americium-241 there are over a 125 million households in the US with most of them having at least one smoke detector. ${ }^{5}$

\subsubsection{Molecular imaging}

For the class of detectors used here, the goal is to use a radioisotope to "label" a molecule of biological interest. The radioisotope is either a constituent element of the molecule (a radiotracer) such as carbon-11 in ${ }^{11} \mathrm{CO}_{2}$ or the molecule is tagged via the chemical attachment of the radioisotope (radiolabel) such as through iodination. The tagged molecules are then introduced in vivo into biological systems: tissue samples, people, animals, plants and microbes. The tagged molecules are detected or "imaged" ex vivo as with autoradiography or externally in vivo through the use of detector technology derived from nuclear particle physics detectors. The presence, amount and extent, or biodistribution of tagged molecules is imaged by devices capable of detecting the emitted particles. Beta particles or low energy x-rays can be detected with autoradiography or high energy photons that are highly penetrating can be detected and imaged externally using the nuclear medicine imaging systems such as gamma cameras, SPECT and PET.

In biomedical applications such as in clinical diagnostics and preclinical research radiotracers/radiolabeling is extremely sensitive, approaching tens of molecules needed for detection. Though one needs ten thousand to be imaged this still represents trace 
amounts that have a low probability of biochemically perturbing the biological system. The availability of an array of radiotracers with different half-lives and emitted radiation and chemical compounds to chose provide many options to achieve the bio-medical task. Often repeated measurements with the same subject (patient, animal or plant) are possible due to short half-lives. This form of imaging referred to as "functional" imaging as it allows the user to observe the biological function of the labeled molecule. This functional imaging modality can be combined with a structural imaging modality such as $\mathrm{x}$-ray or magnetic resonance imaging.

With radiotracer imaging in medicine or animal based research the patient or animal is injected with a small amount of a radiopharmaceutical (i.e. Tc99m-sestamibi). The compound because of its use in a biological function localizes in a patient (i.e. a tumor) according to the compound's metabolic properties. For instance Tc99m-sestamibi will accumulate in living cells that have an abundance of mitochondria, a cellular component. This is often the case for many types of cancer cells. Mitochondria produce the metabolic energy molecule adenosine triphosphate. Since cancer cells are in a rapid growth phase they have a high caloric need thus a surplus of mitochondria. A gamma-ray of energy 140 $\mathrm{keV}$ is emitted when Tc99m decays. This gamma-ray easily exits the subject and is imaged via a gamma camera or with three dimensional imaging using SPECT detectors. Detectors are mostly based on crystal scintillators and sometimes semiconductors. With PET imaging the radiopharmaceutical is a positron emitter such as F18fluorodeoxyglucose. The emitted positron interacts with a nearby electron giving rise to a pair of simultaneous $511 \mathrm{keV}$ annihilation photons traveling 180 degrees from each other. The annihilation photons are then detected by the PET detectors typically based on crystal scintillators. Both SPECT and PET are well established clinical diagnostic techniques with over ten million studies occurring annually in the US. ${ }^{6}$

\subsection{Accelerator technology accelerating particles to modify a target material}

This final category of applications is heavily represented in the industrial manufacturing arena but has also made a significant impact in healthcare. Here accelerator technology is used to accelerate particles such as electrons, protons or ions to induce a physical, chemical or biological change in the material. Some of the most common uses of accelerated particles include: electron beam processing, ion implantation for semiconductors and hardening; and external beam medical therapy (photons, electrons and protons). Though electron beam processing has found many niche applications such as welding, machining and waste treatment; ion implantation and the medical use of beams have provided more widespread impact and thus I will focus on those.

\subsubsection{Ion implantation}

Ion beams have been used to enhance the surface hardness of metal and polymer. For instance, ion beams have been used to improve the coefficient of friction leading to high performance metal bearings. Perhaps the most significant impact on society is the application of ion beams in the semiconductor industry. It is through the ability to make 
highly sophisticated compact ion doped semiconductor based integrated circuits (IC) that the impact of the application of ion beams is so visible to the public. ${ }^{7}$

In the 1970s ion beams made it possible to fabricate complementary metal-oxide semiconductor transistors that are the dominant form of IC devices such as logic and memory ICs. Advanced computation capabilities and global communications using semiconductor technology are made possible through pioneering work in accelerator nuclear physics. Semiconductors make it possible to have both laser diodes and light sensing photodiodes. These are key technologies in the fiber optic based electron optical systems around which is built much of global communications. Ion implantation has also made it possible to fabricate charge CCDs, semiconductor imaging devices. Photovoltaic (PV) technology is also dependent on ion implantation advances. Though present standard PV manufacturing rely on chemical vapor deposition in the doping process, advanced silicon-based PV development is based on conventional ion.

Conventional ion implantation and plasma immersion implantation: Ions are implanted at doses ranging from $\sim 5 \times 10^{10}$ to $1 \times 10^{19}$ atoms $/ \mathrm{cm}^{2}(\sim \mu \mathrm{A}$ to $100 \mathrm{~mA})$ at energies ranging from $\sim 100 \mathrm{keV}$ to $\sim 10 \mathrm{MeV}$ using either conventional ion implantation (CII) or plasma immersion implantation (PIII). The basic sub-systems of a CII system have their origin in nuclear physics particle accelerator apparatus. The process starts with an ion source that is either a gas of the dopant or generated by heating an element containing the desired element to implant. A plasma is generated and the ions are accelerated to a separating magnet that separates the ions through a slit sweeping away unwanted ions. The ion beam passes through focusing magnets and high voltage acceleration achieves the desired ion implantation energy. The beam is directed to a magnet to select the final ion energy. Incident beam energies range from $\sim 100 \mathrm{eV}$ to $\sim 10$ $\mathrm{MeV}$. Finally, the clean ion beam can be focused and scanned across the silicon wafer. This works well for largely planar objects to be processed. Ion doses peak at $\sim 1 \times 10^{4} \mu \mathrm{A}$

PIII has more recently been developed which can be used when low energy ion implantation and high doses (currents) are desirable. PIII like CI also starts with the process of generating a plasma. However, ion implantation with PIII is achieved by introducing the object (i.e. semiconductor) to receive the ion implant in a plasma of the desired ions and then delivering a pulsed high voltage bias to the object thus accelerating ions into the surface of the object. PIII cannot achieve the high energies needed for all ion implant applications but can reach high doses and implant easily over a range of object shapes as compared to CI.

Ion implantation manufactured integrated circuits: Silicon is the semiconductor of choice for the manufacturing of ICs. Raw silicon is a semiconductor: neither a perfect insulator nor a perfect conductor. Inserting a smattering of boron or phosphorus atoms into the silicon crystal lattice allows for the control of the flow of electricity through the silicon and make transistors - the building block of ICs. In a silicon crystalline lattice there are 4 valence electrons. By substituting different dopant atoms into the crystal one can add charge carries. A P-type dopant such as boron generates a charge acceptor (or hole) and $\mathrm{N}$ - type dopants such as phosphorus adds an electron as a charge carrier. 


\subsubsection{External beam radiation therapy}

External beam radiation therapy uses externally generated beams of photons or charged particles that ionize cancer tissue. Other forms of ionizing radiation therapy also include the introduction of radioactive sources into the body (called brachytherapy) with the resultant emitted ionizing radiation used to treat a disease in situ. Approximately 470,000 patients have received some sort of radiation therapy in the United States per year. ${ }^{8}$ Cancer therapy has benefited significantly from nuclear physics and from the development of particle accelerators. External beam therapies are used to treat cancerous tumors not easily treated with surgery or chemotherapy. The DNA of cells (both cancerous and healthy) is damaged by ionizing radiation leading to cell death. Often radiation therapy is used in combination with chemotherapy and surgery. Therapies have been developed using electron accelerators to produce beams with energies of $\sim 1 \mathrm{MeV}$ photons (x-rays) and proton (hadron) accelerators to achieve proton beam energies $\sim 200$ $\mathrm{MeV} .{ }^{9}$ The goal of radiation therapy is to deposit energy at lethal doses to the tumor while minimizing or eliminating a dose to healthy tissue injury.

$X$-ray and electron beams: Most external beam therapy system make use of electron linacs to either direct a beam of electrons at a patient or at a target such as tungsten to produce a beam of high energy photons (x-rays). Electron beams are used to treat shallow cancers while $\mathrm{x}$-ray beams are used for tumors deeper in the patients body. X-ray beam therapy is most often used. X-ray beam therapy deposits energy (thus potential tissue damage) along the entire path through the patient such that most of the dose is delivered in front of the tumor. To minimize accumulated dose through healthy tissue the total beam therapy is delivered at fractional doses at different entry directions to the tumor sometimes over several days. There are several commercial sources of clinical linacs which have become compact and several thousand have been installed worldwide.

Proton beams: In 1946 Robert Wilson suggested using protons for providing a lethal dose to cancer cells. ${ }^{10}$ With proton therapy the kinetic energy of the proton is mostly deposited or peaks at one depth near the end of its range. This is referred to as the Bragg peak and the therapy concept is explained very completely in a review of proton therapy published in 1995. ${ }^{11}$ This class of therapy which makes use of the Bragg peak also include other ions besides protons such as carbon. The term ion therapy or hadron therapy is often used in this case. The Bragg peak associated with hadron therapy indicates a deposition of more energy in a narrower peak than with proton therapy. This may have some advantages over proton therapy for some cancers but also adds the need to know very accurately where the dose is delivered to avoid damaging health tissue. Presently in the United States there are about 25 proton therapy centers but no hadron therapy centers. There are approximately 35 proton therapy centers in Europe, Asia and Africa and about an additional ten hadron centers using carbon ions. Synchrotrons are the accelerators that are used in proton or hadron therapy to achieve the particle energies. Synchrotrons are significantly more expensive and more massive accelerators than the linacs used in $\mathrm{x}$-ray therapy. 


\section{Summary}

Beyond revealing the nature of the physical universe, nuclear physics clearly has provided and continues to provide society with significant benefits. Curiosity that drives certain individuals to strive to uncover the mysteries of the physical universe is a scarce global resource. It is incumbent on nuclear physicists to be aware of the diversity of the applications of nuclear physics and to communicate the impact that their discipline has had to our way of life.

\section{Acknowledgments}

I am grateful to Dr. Carl Zorn for his careful reading of the manuscript and his many helpful suggestions. Authored by Jefferson Science Associates, LLC under U.S. DOE Contract No. DE-AC05-06OR23177.

\section{References}

1. Industrial Accelerators and Their Applications eds. R Hamm and M. E. Hamm (R \& M Technical Enterprises, California, 2012).

2. Reviews of Accelerator Science and Technology, vol 4 (World Scientific, 2011).

3. Nuclear Physics: Exploring the Heart of Matter, Committee on the Assessment of and Outlook for Nuclear Physics, National Academy of Sciences, (National Academies Press, Washington, DC, 2013).

4. K. Kostecka, Americium- from discovery to the smoke detector and beyond, Bull. Hist. Chem., vol 33, n. 2, (2008).

5. M. Aherns, Smoke Alarms in U.S. Home Fires, National Fire Protection Association Report (September 2015).

6. Emission Tomography: The Fundamentals of PET and SPECT, eds.: M. N. Wernick and J. N. Aarsvold, (Academic Press, San Diego, 2004).

7. L. A. Larson \& J.M. Williams, Ion Implantation for Semiconductors Doping and Materials Modification, in Reviews of Accelerator Science and Technology, vol 4 (World Scientific, 2011), p. 11.

8. D.E Citrin, Recent Developments in Radiotherapy, N Engl J Med 377 (Sept. 14, 2017) p. 1065 .

9. B.R. Martin, in Nuclear Particle Physics, 2nd edn, (Wiley and Son, West Sussex, 2012) p. 282.

10. R.R. Wilson, Radiological uses of fast protons, Radiology, 47, 487 (1946).

11. D.W. Miller, A review of proton beam radiation therapy, Medical Physics, 22, 1943 (1995). 